\title{
Analytical Mechanics and Almost Vibrationally-Adiabatic Chemical Reactions
}

\author{
By R. A. MARcus \\ Noyes Chemical Laboratory, University of Illinois, Urbana, \\ Illinois 61801, U.S.A.
}

Received 14th June 1967

Co-ordinates and "vibrationally-adiabatic" approximations are described for reactions in three dimensions. Some reactions may demonstrate a fairly strict adiabaticity for certain degrees of freedom and a statistical adiabaticity for others. Some will simply be strongly vibrationally-nonadiabatic. Several topics in kinetics are considered from the viewpoint of vibrational-adiabaticity or mild non-adiabaticity.

\section{1.-INTRODUCTION}

To treat the mechanics of chemical reactions it is desirable to have a set of co-ordinates (natural collision co-ordinates) which pass smoothly from those suited to reactants to those suited to products. Such a set was recently given for linear collisions ${ }^{1}$ and an approximate solution was obtained for nearly vibrationallyadiabatic reactions. A recent extension to three dimensions ${ }^{2}$ is summarized below, and the probable nature of several nearly vibrationally-adiabatic solutions is outlined. In anticipation of the results of such a mode of solution, several problems are examined: translation-vibration interaction in chemically-reactive collisions, mechanics and a statistical-dynamical theory of reaction cross-sections, ${ }^{3}$ quantum corrections to computer trajectories, and the quasi-equilibrium assumption in kinetics.

\section{2.-NATURAL COLLISION CO-ORDINATES}

In a three-centre reaction, ${ }^{4}$

$$
\mathrm{AB}+\mathrm{C} \rightarrow \mathrm{A}+\mathrm{BC}
$$

there are six-co-ordinates in the centre-of-mass system. On introducing body-fixed co-ordinates via an Eulerian angle $(\theta, \phi, \chi)$ rotation matrix ${ }^{5}$ and then letting the body-fixed $y z$ plane be the instantaneous plane of the three atoms, there are obtained seven co-ordinates of which one is redundant. This seventh is eliminated by defining the orientation of the body-fixed axes in the $y z$ plane. A locally Cartesian method was used. ${ }^{6}$

Curvilinear co-ordinates $n$ and $s$ were introduced ${ }^{1}$ to replace $z$ and $Z$, as in fig. 1 . (Here, $z$ is a scaled ${ }^{7} z$-component of the $\mathrm{AB}$ distance and $Z$ is a scaled $z$-component of the distance between $\mathrm{C}$ and the centre of mass of AB.) A co-ordinate $m$ of magnitude $\left(y^{2}+Y^{2}\right)^{\frac{1}{2}}$ was then defined. ${ }^{8}$ It describes the nonlinearity of the configurations.

The six independent co-ordinates are now $s, n, m, \theta, \phi, \chi$. Potential energy profiles in the $(m, n, s)$ subspace have cross-sectional shapes indicated by the shaded regions in fig. 2. The reacting system typically moves through these regions. For this reason, co-ordinates $(m, n)$ were transformed into polar co-ordinates $(r, \gamma)$, where $m$ and $n$ equal $r \sin \gamma$ and $r_{0}-r \cos \gamma, r_{0}$ being determined by the shape of the profiles and being a function of $s$, the reaction co-ordinate.

Finally, to remove bothersome cross-terms at large $\pm s$ between $\dot{\gamma}$ and $(\dot{\theta}, \dot{\phi}, \dot{\chi})$ 
the angles $\gamma$ and $\chi$ can be transformed into angles describing orientation with respect to space-fixed axes : a matrix $(\sin \Theta \cos \Phi, \sin \Theta \sin \Phi, \cos \Theta)$ was expressed in terms of $(0, \sin \gamma, \cos \gamma)$ by the Eulerian angle rotation matrix. (However, for some purposes, co-ordinates $\mathrm{s}, \mathrm{r}, \gamma, \theta, \phi, \chi$ may be more convenient than $\mathrm{s}, \mathrm{r}, \Theta, \Phi, \theta, \phi$.

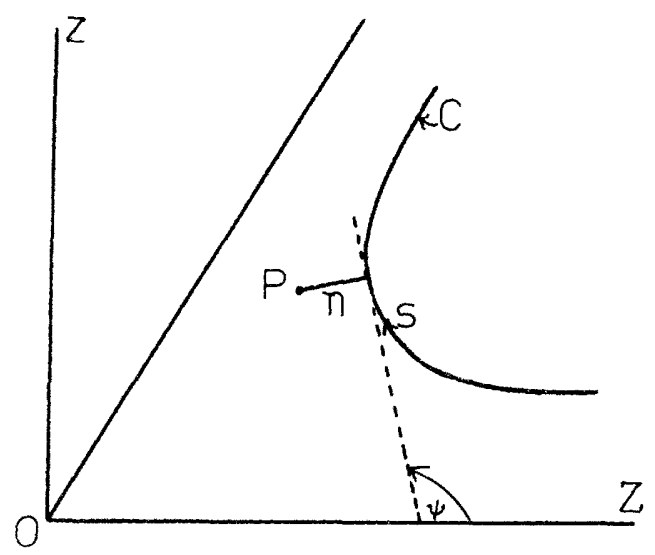

Fig. 1.-Orthogonal curvilinear co-ordinates $(n, s)$ of any point $\mathrm{P} . \quad n$ is the perpendicular distance to any curve $\mathrm{C}$ and $s$ is the distance from any fixed point on $\mathrm{C}$ to the foot of that perpendicular.

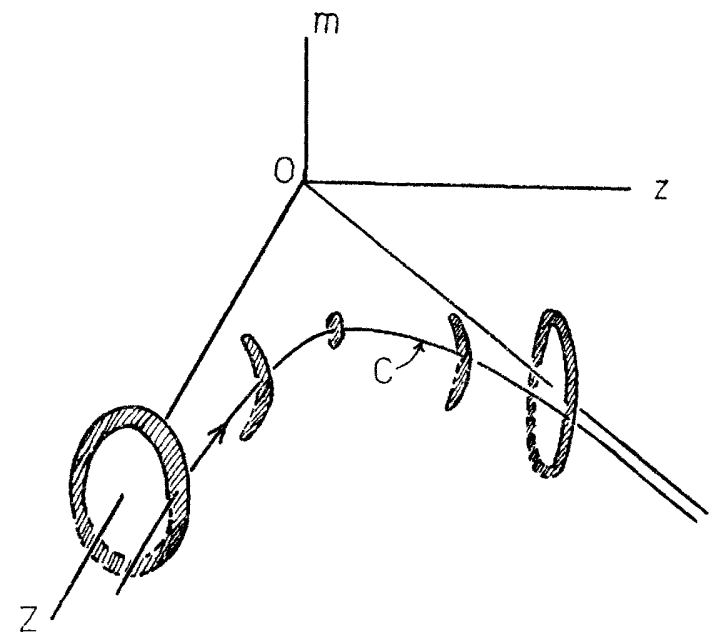

Fig. 2.-Cross-sections of a potential energy surface. The $Z z$ plane, curve $C$, and skewed axes are those of fig. 1. A reacting system moves throught the shaded regions, executing a rotation (free at first), a vibration and a translation in this $(m . n, s)$ internal co-ordinate space. Initially, the rotation is about the $\mathrm{OZ}$ axis, the vibration is perpendicular to $\mathrm{OZ}$ and the translation is parallel to $\mathrm{OZ}$.

The potential energy is a relatively simple function of $(s, r, \gamma){ }^{9}$ The Hamiltonian is of the form given by $(2 \cdot 2)$, where $T_{c}$ denotes a number of cross-terms, of which those between the two sets of angle variables, $(\theta, \phi)$ and $(\Theta, \Phi)$, are most prominent. These cross terms vanish when $s \cong \pm \infty$.

$$
H \cong \frac{1}{2 \mu}\left(p_{r}^{2}+\frac{p_{s}^{2}}{\eta^{2}}\right)+\frac{1}{2 \mu r_{0}^{2}}\left(p_{\Theta}^{2}+\frac{p_{\Phi}^{2}}{\sin ^{2} \Theta}\right)+\frac{1}{2 I}\left(p_{\theta}^{2}+\frac{p_{\phi}^{2}}{\sin ^{2} \theta}\right)+T_{c}+V .
$$

Here, $\mu$ is $\left[m_{A} m_{B} m_{C} /\left(m_{A}+m_{B}+m_{C}\right)^{\frac{1}{2}}, \eta\right.$ is essentially $1+\left(r_{0}-r\right) \kappa, \kappa(s)$ is the curvature of curve $\mathrm{C}$ in fig. 1 at any $s$, and $I(s)$ is essentially the largest moment of inertia at each $s$. 
The potential energy $V$ can be written as the value $V_{1}(s)$ for any point on curve $C$ plus the increment $V_{2}(s, r, \gamma)$ to go to any other point in the $(m, n, s)$ space at the same $s$. If the $r$-motion is assumed to be coupled mainly to the $s$-motion, (approximation 3 of $\S 3$ ) then

$$
V \cong V_{1}(s)+V_{2}\left(s, r_{0}, \gamma\right)+V_{2}(s, r, 0)-V_{2}\left(s, r_{0}, 0\right) .
$$

TABLE 1.-Physical NATURE OF THE CO-ORDINATES FOR SEVERAL VAlues OF REACTION CO-ORDINATE $s$

\begin{tabular}{|c|c|c|c|}
\hline co-ordinate & nature at $s \simeq-\infty$ & nature at $s \simeq s^{\neq}$ & nature at $s \simeq+\infty$ \\
\hline$s$ & $\begin{array}{l}\text { radial translation of } \\
\mathrm{AB} \text { relative to } \mathrm{C}\end{array}$ & $\begin{array}{l}\text { asym. stretching vibra- } \\
\text { tion of } A B C \neq\end{array}$ & $\begin{array}{l}\text { radial translation of } \mathrm{A} \\
\text { relative to } \mathrm{BC}\end{array}$ \\
\hline$r$ & vibration of $\mathrm{AB}$ & $\begin{array}{l}\text { sym. stretching vibra- } \\
\text { tion of } \mathrm{ABC} \neq\end{array}$ & vibratio \\
\hline & $\begin{array}{l}\text { orbital translation of } \\
\mathrm{AB} \text { relative to } \mathrm{C}\end{array}$ & & $\begin{array}{l}\text { orbital translation of } \mathrm{A} \\
\text { relative to } \mathrm{BC}\end{array}$ \\
\hline$\Theta, \Phi$ & rotation of $\mathrm{AB}$ & $\begin{array}{l}\text { doubly degenerate ben- } \\
\text { ding vibration of } A B C \neq\end{array}$ & rotations of $\mathrm{BC}$ \\
\hline
\end{tabular}

$a$ This nature applies only the adiabatic case $\mathrm{A}$ in the text. For adiabatic case B these two items would be interchanged.

$b \Theta-\theta$ and $\Phi-\phi$ describe the bending.

The physical significance of the co-ordinates, in various regions of the sixdimensional configuration space, is given in table 1 . At values of $s$ intermediate between large $\pm s$ and $s^{\neq}$, the $\Theta, \Phi$ motions are hindered rotations rather than rotations or bending vibrations.

\section{3.- SOLUTION OF THE EQUATIONS OF MOTION}

The Hamilton-Jacobi and Schrodinger partial differential equations are obtained from a Hamiltonian such as (2.2) in the standard way. ${ }^{10,11}$ Because of the close relationship between the two partial differential equations, parallel treatments of the classical and quantum mechanics can be made. As the extensive literature on collisions and on molecular vibration-rotation interactions amply testifies, there is a variety of subsequent treatments of these equations which can be introduced. An adiabatic-nonadiabatic approach is perhaps the most tractable. One adiabatic version assumes $^{2}$ : (1) all motions are adiabatic relative to the motion along $s$, the r-motion being the most adiabatic of all ; (2) Case $A$ the $\Theta, \Phi$ motion is adiabatic relative to the $\theta, \phi$ one, or Case $B$ the $\theta, \phi$ motion is adiabatic relative to the $\Theta, \Phi$ one;

(3) apparently minor $r, \gamma$ coupling terms can be neglected in the potential energy.

Because of the assumed adiabaticity of the $r$-motion especially, approximation (3) could be fairly easily avoided, but it does lead to simpler results. Approximations (1) and (2) are made classically and quantum mechanically by writing (for case A, for example)

$$
\begin{gathered}
\psi \cong \psi_{1}(s) \psi_{2}(r ; s) \psi_{3}(\Theta, \Phi ; \theta, \phi, s) \psi_{4}(\theta, \phi ; s) \\
W \cong W_{1}(s)+W_{2}(r ; s)+W_{3}(\Theta, \Phi ; \theta, \phi, s)+W_{4}(\theta, \phi ; s),
\end{gathered}
$$

where $W$ is Hamilton's characteristic function. The adiabatic approximation is actually made by neglecting the partial derivatives of each $\psi_{i}$ or $W_{i}$ with respect to the variables to the right of the semicolon, which we shall call its weakly-dependent variables. In case $\mathrm{B}$, the $\Theta, \Phi$ and the $\theta, \phi$ in the above equations are interchanged.

In the adiabatic approximation, variations in weakly-dependent variables of a $\psi_{i}$ (or $W_{i}$ ) do not change the quantum numbers (or classical phase integrals) associated with it. Neverthless, in this adiabatic approximation large changes may 
occur in the physical nature of the strongly-dependent degrees of freedom (viz., table 1), in the shape of $\psi_{i}$ or $W_{i}$, and in the energy associated with the $\psi_{i}$ or $W_{i}$. (One obtains, in fact, adiabatic correlation diagrams for the energy contributions.)

In the next approximation the various approximations (1)-(3) can be examined. For example, (3) and others not mentioned can be examined by perturbation methods. The non-adiabatic corrections to (1) and (2) can be studied in several ways, one approximate one being of particular interest. It is relatively straightforward, maintains the parallelism of the classical and quantum calculations, and has intuitive appeal : the weakly-dependent parameters in any $\psi_{i}$ or $W_{i}$ are replaced by the classical time-dependent solutions of the adiabatic equations, and the time-independent Schrodinger and Hamilton-Jacobi equations for a $\psi_{l}$ and a $W_{l}$ are replaced by the corresponding time-dependent ones.

In this way it is possible to calculate approximately the extent of excitation of the vibrational, rotational and orbital motions of the products. In this time-dependent treatment of non-adiabatic calculations of cases A and B can be regarded as the chemical counterparts of the low mass and flywheel approximations, respectively. The latter were introduced by Cross and Herschbach ${ }^{12}$ in their calculation of classical rotational-translational energy exchange. Moreover, the adiabatic collision in the chemical reaction case can be regarded as the analogue of the elastic collision in physical scattering: in both cases some event happens (e.g., reaction or scattering), but there is no change in quantum numbers or classical phase integrals of the periodic motions.

When a considerable change of reduced mass occurs, as, for example, in

$$
\mathrm{H}+\mathrm{Cl}_{2} \rightarrow \mathrm{HCl}+\mathrm{Cl} \text {, }
$$

one limiting approximation might prevail before the activated complex region $\left(s \leqslant s^{\neq}\right)$and the other one after $\left(s \geqslant s^{\neq}\right)$. In (3.3) the flywheel analogue could prevail initially and the low mass one finally.

\section{4.-VIBRATION-REACTION CO-ORDINATE INTERACTIONS}

It appears that the present equations for the $r$, $s$ motions can be made similar to those derived for linear collisions elsewhere, ${ }^{1}$ so that analogous deductions would then follow. Several are summarized below.

CURVILINEAR TUNNELLING.- The standard method of computing tunnelling rates in chemical reactions calculates the barrier along the "reaction path" and assumes a one-dimensional Cartesian co-ordinate kinetic energy. It actually over-estimates the tunnelling rate for any given potential energy surface thereby. ${ }^{1}$ The error is small with energies just below the top of the barrier, but increases as the energy difference becomes increasingly negative. Two-dimensional calculations based on natural collision co-ordinates $r$ and $s$ have been used ${ }^{1}$ to estimate the correct tunnelling rate, and may explain overestimates of tunnelling rates by the standard method. Detailed comparison with computer results would be of interest.

CONTRIBUTION OF VIBRATION TO RATE.-If the vibration frequencies of $A B$ and (symmetric stretching) of $\mathrm{ABC}^{\neq}$are $v$ and $v^{\neq}$, the adiabatic change of vibrational energy is $\left(v+\frac{1}{2}\right) h\left(v^{\neq}-v\right)$ (quantum), or $J_{v}\left(v^{\neq}-v\right)$ (classical), where $J_{v}$ is $\oint p_{r} \mathrm{~d} r$, the phase integral for the vibrational motion. ${ }^{13}$ Typically, $v^{\neq}<v$, causing a decrease in vibrational energy. According to the present $r, s$ equations, the liberated energy goes into the energy of the $s$-motion, and so helps the system overcome the barrier by that amount. The agreement of this result with the results of numerical classical mechanical integrations for linear collisions has been discussed previously. ${ }^{13}$ 
VIBRATION EXCITATION OF PRODUCTS.-In addition to adiabatic changes of vibrational energy non-adiabatic changes can also occur, corresponding to changes in vibrational quantum number or phase integral. A comparison of the calculated results with computer data will be interesting. The theory should break down when the non-adiabatic effects become too large.

\section{5.-RELATION TO A STATISTICAL-DYNAMICAL THEORY OF REACTION CROSS-SECTIONS}

The earlier analytical mechanical discussion indicates that for any given impact parameter and initial relative velocity of the colliding pair the barrier to reaction is several-fold: (1) the potential energy barrier; (2) the centrifugal potential energy barrier, which is calculated in a simple way when approximate adiabaticity of the co-ordinate associated with the orbital angular momentum prevails ; (3) the change of vibrational energy in forming the activated complex in the given vibrational state for the $r$-motion; and (4) the increment in energy when the transient bending modes are formed from the initial rotational ones (states for bending modes are more widely spaced than those for the rotations).

In a recent statistical-dynamical theory ${ }^{3}$ of these reactions, simple expressions were obtained for the total reaction cross-section and for the reaction probability at any impact parameter, as a function of initial rotational, vibrational, and translational state of the reactants. In the theory the above four contributions to the effective barrier were taken into account, the fourth in a statistically-adiabatic manner such that the quasi-equilibrium assumption in $\$ 7$ was satisfied. Detailed classical computer data have been given for the reaction cross-sections of the $\mathrm{H}+\mathrm{H}_{2}$ reaction. ${ }^{14}$ In a comparison of the theory with these data the agreement between them was encouraging, there being no adjustable parameters. ${ }^{3}$ At high translational energies the comparison indicated occurrence of some vibrational non-adiabaticity. With the aid of an approximate solution for the present equation for the $s$-motion it would be possible to extend this simple model to estimate differential reaction cross-sections.

\section{6.-QUANTUM CORRECTIONS FOR CLASSICAL TRAJECTORIES}

Reaction cross-sections have been obtained from computer data only for classical mechanical systems. Some quantum correction of the results for the $\mathrm{H}+\mathrm{H}_{2}$ reaction was made ${ }^{14}$ by restricting the initial rotational-vibrational energy to values allowed by quantum mechanics. This point is now explored further.

At any value of the reaction co-ordinate $s$ before the system reacts, some quantum corrections can be made via the WKB method. Namely, the initial vibrational, rotational, orbital, $z$-component of rotational and $z$-component of orbital phase integrals $\oint p_{i} \mathrm{~d} q^{i}$ are set equal to the values $\left(v+\frac{1}{2}\right) h,\left(j+\frac{1}{2}\right) h,\left(l+\frac{1}{2}\right) h, m_{j} h, m_{l} \boldsymbol{h}$, respectively. ${ }^{15}$ (These refer to the $r,(\Theta, \Phi),(\theta, \phi), \Phi$ and $\phi$ motions, respectively.) If the adiabaticity occurred at later values of $s$, i.e., if the equations were adiabatically separable into onedimensional equations for all $s$, the above restrictions on the phase integral would be automatically imposed by virtue of their having been imposed initially.

The procedure of only restricting the initial rotational-vibrational energies to those allowed by quantum mechanics amounts to imposing two of the above five phase integral conditions. Because of the possible adiabatic correlation of the original orbital-rotational motion of the reactants with the rotational-bending vibrational motion of the activated complex, it is desirable to impose the other phase integral conditions to achieve better results for the threshold region. Otherwise, 
any zero-point, bending vibrational motion (if it indeed occurs) would be missed. However, outside of the threshold region the procedure originally used ${ }^{14}$ should suffice.

As application of the WKB method to the adiabatically-separated equations shows, there are additional quantum effects influencing the motion along the reaction co-ordinate (e.g., diffraction). However, they are less important for the reaction rate, except at low enough energies for tunnelling to occur.

A further effort towards computing quantum effects might be made by comparing the classical mechanical computer data of the original equations with those based on equations involving natural collision co-ordinates and into which various simplifying approximations have been introduced. If suitable agreement is achieved, computer studies of the quantum analogues of the latter would be useful.

7.-QUASI-EQUILIBRIUM, ADIABATICITY AND STATISTICAL ADIABATICITY

In the present section a quasi-equilibrium relation between reactants and activated complexes of the same energy $E$ is derived assuming adiabaticity. ${ }^{16}$ The resulting quasi-equilibrium equation has been tested ${ }^{17}$ by comparison with the computer data. The results are summarized later.

We consider a reacting pair whose total energy lies in $(E, E+\mathrm{d} E)$ and which is in a quantum state $v$ for certain vibrational modes. The quantum numbers of the other vibrational and rotational modes are denoted by $n$. The remaining quantum numbers are $l$ and $m_{l}$, where $l$ describes the initial orbital angular momentum. If the initial momentum along the reaction co-ordinate $q$ is $p$ and the initial energy of the rotationalvibrational modes of the reacting pairs in $E_{l v n}$, then

$$
E=E_{\mathrm{lvn}}+p^{2} / 2 \mu
$$

where $E_{l v n}$ is independent of $l$. The number of translational states of a pair in state $l v n$, in $(E, E+\mathrm{d} E)$ and in $(q, q+\mathrm{d} q)$ is $\mathrm{d} p \mathrm{~d} q / \boldsymbol{h}$, where $p \mathrm{~d} p / \mu$ is $\mathrm{d} E$. The reactive flux of such states, computed by dividing by $\mathrm{d} q$ to obtain a density along $q$ and by multiplying by $\dot{q}$ and by the reaction probability $w_{l v n p}$, is $w_{l v n} \mathrm{~d} E / \boldsymbol{h}$, since $\mathrm{d} E$ is $\dot{q} \mathrm{~d} p$. The reactive flux from all states in $(E, E+\mathrm{d} E)$ is $\mathrm{d} E \sum_{n}(2 l+1) w_{l v n p} / \boldsymbol{h}$. The sum is over all $n$ for which $E_{t v n} \leqq E$.

A state $l v n$ adiabatically connects with one whose energy for all rotationalvibrational modes in the activated complex region is $E_{l v n}^{\neq}$. $E$ now equals $E_{l v n}^{\neq}+p_{\neq}^{2} / 2 \mu_{\neq}$, where the second term is the kinetic energy along the reaction co-ordinate. Of the original states, those which lead to reaction are those for which $E_{l v n}^{\neq} \leqslant E$. The number of states $l v n$ in $\mathrm{d} q^{\neq}$and in $\mathrm{d} E$ is $\mathrm{d} q^{\neq} \mathrm{d} p_{\neq} / \boldsymbol{h}$, where $\mathrm{d} p_{\neq}=\mu_{\neq} \mathrm{d} E / p_{\neq}$. The flux is $\dot{q}^{\neq} \mathrm{d} p_{\neq} / \boldsymbol{h}$ and so is $\mathrm{d} E / \boldsymbol{h}$. An adiabatic noncrossing rule is now invoked, and thereby this flux expression is summed over all states for which $E_{l v n}^{\neq} \leqslant E$ and over all reaction paths if there is more than one. Thus,

$$
\begin{array}{cc}
\sum_{l n}(2 l+1) w_{l v n p} & =\Gamma \sum_{m_{l} l n} 1 \\
\left(E_{l v n} \leqslant E\right) & \left(E_{l v n}^{\neq} \leqslant E\right)
\end{array}
$$

where $\Gamma$ is a summation operator over all reaction paths. To avoid confusion we replace $m_{l} l n$ on the right side by $n^{\neq}$. (The designation $m_{l} l n$ merely indicates origin of a state in a correlation diagram.) Analysis by separation into states of different $J$, leads to eqn. (5) of ref. (17) and thence to (7.2).

Eqn. (7.2) is a quantitative statement of a quasi-equilibrium between reacting pairs and activated complexes of the same $E$ moving in the forward direction and was originally derived ${ }^{16}$ in that way. From it, an activated complex theory rate expression can also be derived if an equilibrium distribution of reactants' states is assumed. 
In terms of reaction cross-sections $\sigma_{v n p},(7.2)$ becomes

$$
\sum_{n}\left(k^{2} / \pi\right) \sigma_{v n p}=\Gamma \sum_{n \neq} 1
$$

since $\sigma_{v n p}$ equals $^{18}\left(\pi / k^{2}\right) \sum_{l=0}^{\infty}(2 l+1) w_{l v n p}$. In (7.3), $k$ denotes $p / \hbar$.

In a test of (7.3) using computer data and classical versions of the sums, the lefthand side was found to be $9 \cdot 6,24.5$ and 55 , while the right-side was $7 \cdot 0,22$ and 50 , when $E$ was $15.5,17.0$ and $18.5 \mathrm{kcal} / \mathrm{mole}$, respectively. ${ }^{17,19}$ The agreement is encouraging. ${ }^{20}$ The $E$ are those of interest in thermal reaction studies.

However, one might expect the rotational and orbital modes to be adiabatic in at best a statistical sense rather than a rigorous one. This statistical adiabaticity would prevail when each relevant initial state yields at each $s$ not necessarily a single state but a group of states fairly symmetrically distributed in energy about the adiabatically determined state at that $s$. It will be interesting to see if the computer data exhibit a statistical adiabaticity for those modes and to see when the quasi-equilibrium relation (7.3) can be derived from a suitable statistical adiabaticity.

Acknowledgement is made to the donors of the Petroleum Research Fund, administered by the American Chemical Society, and to the National Science Foundation, for their support of this research.

${ }^{1}$ R. A. Marcus, J. Chem. Physics, 1966, 45, 4493, 4500.

${ }^{2}$ R. A. Marcus, to be published.

3 R. A. Marcus, J. Chem. Physics, 1966, 45, 2630; 1967, 46, 959.

4 A reaction path which forms AC may also occur and would have its own set of natural collision co-ordinates. ${ }^{2}$

${ }^{5}$ E. B. Wilson, Jr., J. C. Decius and P. C. Cross, Molecular Vibrations (McGraw-Hill, New York 1955), p. 286.

${ }^{6}$ In the subsequent notation, the orientation is altered until $y \sin \zeta=-Y \cos \zeta$, where $\zeta(s)$ is chosen to eliminate one of the vibrational angular momentum terms at each $s$. $\zeta=\psi$ in fig. 1 when $s \cong \pm \infty$.

7 The scaling factors in F. T. Smith, J. Chem. Physics, 1959, 31, 1353, are used.

${ }^{8} y=-m \cos \zeta(s), Y=m \sin \zeta(s)$.

${ }^{9} \gamma$ is related to $\theta$, $\Phi$ etc., via $\cos \gamma=\cos \theta \cos \Theta+\sin \theta \sin \Theta \cos (\varphi-\Phi)$.

10 e.g., H. Goldstein, Classical Mechanics (Addison-Wesley, Reading, Mass., 1950), pp. $280 \mathrm{ff}$.

11 e.g. W. Pauli, Jr., in Handbuch der Physik., S. Flügge, ed. (Springer-Verlag, Berlin, 1958), vol. 5 , p. 39.

12 R. J. Cross, Jr., and D. R. Herschbach, J. Chem. Physics., 1965, 43, 3530.

${ }^{13}$ R. A. Marcus, J. Chem. Physics., 1965, 43, 1598.

${ }_{14}$ M. Karplus, R. N. Porter and R. D. Sharma, J. Chem. Physics, 1965, 43, 3259.

15 R. E. Langer, Physic. Rev., 1937, 51, 669 ; E. C. Kemble, The Fundamental Principles of Quantum Mechanics (Dover, New York, 1958) p. 155.

${ }^{16}$ For simplicity of presentation, certain curvilinear effects are omitted, as is atom tunnelling. The former cause no difficulty and still lead to (7.3), the modified derivation being seen from the arguments leading to eqn. (3) of ref. (17). If tunnelling is also included, (7.3) is replaced by that eqn. (3).

17 R. A. Marcus, J. Chem. Physics, 1965, 45, 2138.

${ }^{18}$ L. D. Landau and E. M. Lifschitz, Quantum Mechanics (Addison-Wesley, Reading, Mass., 1958), p. 537.

${ }^{19}$ A more accurate estimate of the right-side of (7.3) could have been made by taking into account some neglected rotation-vibration interactions.

${ }^{20}$ This agreement differs from the apparent disagreement in ref. (14) of a factor of 6 at $300^{\circ} \mathrm{K}$ in trajectory-calculated and transition-state rate constants. It would be useful to replace the incomplete hybrid quantum-classical comparison in ref. (14) by an all-classical one and by the more complete hybrid proposed in $\$ 6$. Above $1000^{\circ} \mathrm{K}$, incidentally, quantum corrections influenced mainly the stretching vibrations (judging from the partition functions of reactants and activated complex), and the discrepancy in the $k$ was only a factor of $1 \cdot 25$. Accurate tests of the quantum version of transition-state theory require quantum rather than classical computerexperiments. 